hep-ph/0105147

ANL-HEP-PR-01-023

UAHEP013

\title{
Upsilon Decay to a Pair of Bottom Squarks
}

\author{
Edmond L. Berger ${ }^{a}$ and L. Clavelli ${ }^{b}$ \\ ${ }^{a}$ High Energy Physics Division, Argonne National Laboratory \\ Argonne, IL 60439 \\ email: berger@anl.gov \\ ${ }^{b}$ Department of Physics and Astronomy, University of Alabama \\ Tuscaloosa, AL 35487 \\ email: lclavell@bama.ua.edu
}

(May 15, 2001)

\begin{abstract}
We calculate the rate for $\Upsilon$ decay into a pair of bottom squarks as a function of the masses of the bottom squark and the gluino. Data from decays of the $\Upsilon$ states could provide significant new bounds on the existence and masses of these supersymmetric particles.
\end{abstract}


Introduction. The possibility of a light bottom squark, $\widetilde{b}$, with mass of order $10 \mathrm{GeV}$ or less, is examined in several theoretical [1] [0] and experimental [6] papers. If the mass $m_{\tilde{b}}$ is less than half the mass of one of the $J^{P C}=1^{--} \Upsilon$ states, then the decay $\Upsilon \rightarrow \widetilde{b} \bar{b}$ might proceed with sufficient rate for experimental observation or exclusion of a light $\widetilde{b}$. In this paper, we compute the expected rate for $\Upsilon$ decay into a pair of bottom squarks as a function of the masses of the bottom squark and the gluino. The mass of the gluino $\widetilde{g}$ enters because the gluino is exchanged in the decay subprocesses we compute. The bottom squark and the gluino are the supersymmetric partners of the bottom quark and gluon, respectively.

Measurements at $e^{+} e^{-}$colliders do not yet constrain significantly the existence of $\tilde{b}$ 's in the mass region $m_{\tilde{b}}<10 \mathrm{GeV}$ [1]. If a light bottom squark $\left(\widetilde{b}_{1}\right)$ is an appropriate mixture of left-handed $(L)$ and right-handed $(R)$ bottom squarks, its tree-level coupling to the neutral gauge boson $Z$ can be made small by adjustment of the $L-R$ mixing, leading to good agreement with observables at the $Z[2]$. Owing to their $p$-wave coupling to the intermediate photon and fractional charge $-1 / 3$, bottom squarks make a small contribution to the inclusive cross section for $e^{+} e^{-} \rightarrow$ hadrons [6,7], in comparison to the contributions from quark production, and $\tilde{b} \tilde{b}$ resonances are likely to be impossible to extract from backgrounds [3]. The angular distribution of hadronic jets produced in $e^{+} e^{-}$annihilation can be examined in order to bound the contribution of scalar-quark production. Spin-1/2 quarks and spin-0 squarks emerge with different distributions, $\left(1 \pm \cos ^{2} \theta\right)$, respectively. The measured angular distribution [8] is consistent with the production of a single pair of charge- $1 / 3$ squarks along with five flavors of quark-antiquark pairs [1]. The exclusion [9] of a $\tilde{b}$ with mass 3.5 to 4.5 $\mathrm{GeV}$ does not apply since that analysis focuses on decays into leptons, $\tilde{b} \rightarrow c l \tilde{\nu}$ and $\tilde{b} \rightarrow c l$. A long-lived $\widetilde{b}$ or one that decays via baryon-number-violating $R$-parity violating couplings would evade the CLEO limitation [1].

The possibility that the gluino may be much less massive than most other supersymmetric particles is intriguing from different points of view [10-13]. The current status of the very low mass gluino scenario, further possibilities, and more complete references are found in Ref. [12]. The production of a pair of gluinos with mass in the range 12 to $16 \mathrm{GeV}$, decaying with $100 \%$ branching fraction into a bottom quark and a bottom squark in the mass range 2 to $5.5 \mathrm{GeV}$, offers a supersymmetry (SUSY) explanation for the large bottom quark cross section at hadron colliders, and it is consistent with hadron collider data on the timeaveraged $B^{0} \bar{B}^{0}$ mixing parameter $\bar{\chi}[1]$. A renormalization group argument [4] suggests that the existence of a light $\tilde{b}$ goes hand-in-hand with a comparatively light $\tilde{g}$.

To compute $\Upsilon$ decay into a pair of bottom squarks, we follow the established color singlet approach [14 16] in lowest-order perturbative quantum chromodynamics (QCD). We assume that the $\Upsilon$ is a $b \bar{b}$ bound state of bottom quarks and that each $b$ carries half of the momentum of the $\Upsilon$ with $2 m_{b}=m_{\Upsilon}$. Refinements may be considered, but this simple approach is sufficient for the purposes of this first paper on the topic.

Notation and Normalization. Define the four-momentum of the $\Upsilon$ to be $p_{\Upsilon}$. The polarization vector of the $\Upsilon$ is denoted $\epsilon$. The $J^{P}=1^{-}$bound state of the $b$ and $\bar{b}$ quarks is represented as

$$
\frac{1}{\sqrt{2}} \not \frac{\not p_{\Upsilon}+m_{\Upsilon}}{2} A C_{i j},
$$

where the color matrix $C_{i k}=\frac{1}{\sqrt{3}} \delta_{i k}$ expresses the color singlet system of the $b$ with color 
index $i$ and $\bar{b}$ with color index $k$. The coupling strength of the $\Upsilon$ to $b \bar{b}$ is specified in terms of an overall constant $A$ which is related to the value of the orbital wave function at the origin in momentum space [17]. This constant may be expressed in terms of the electronic width $\Gamma_{\ell \bar{\ell}}^{\Upsilon}$ or the hadronic width $\Gamma_{\mathrm{h}}^{\Upsilon}$. In leading order,

$$
\Gamma_{\ell \bar{\ell}}^{\Upsilon}=\frac{8 \pi A^{2} e_{\mathrm{b}}^{2} \alpha_{\mathrm{em}}^{2}}{m_{\Upsilon}}
$$

where $e_{b}=-1 / 3$. The lowest-order perturbative QCD expression for a three-gluon decay width is 17

$$
\frac{\Gamma_{\mathrm{h}}^{\Upsilon}}{\Gamma_{\ell \bar{\ell}}^{\Upsilon}}=\frac{10\left(\pi^{2}-9\right)}{9 \pi} \frac{\alpha_{s}^{3}(\mu)}{\alpha_{\mathrm{em}}^{2}},
$$

where the strong coupling $\alpha_{s}(\mu)$ is evaluated at a momentum scale $\mu \sim m_{\Upsilon}$. However, next-to-leading order contributions are known to be large [18]. In our work, we prefer to use the electronic width as our source of absolute normalization.

The $\Upsilon$ decays into a pair of bottom squarks $\widetilde{b}$ of mass $m_{1}$ carrying four-momenta $k_{1}$ and $k_{2}$ respectively. This decay proceeds via the two-parton to two-sparton subprocess

$$
b+\bar{b} \rightarrow \widetilde{b}+\overline{\widetilde{b}}
$$

with exchange of a $t$-channel gluino $\widetilde{g}$ of mass $m_{\tilde{g}}$. The subprocess is sketched in Fig. 1 . The subprocess in which the $b \bar{b}$ pair annihilates through an intermediate gluon into a $\tilde{b} \bar{b}$ pair is absent because the initial state is a color singlet.

Mixing. The mass eigenstates of the bottom squarks, $\tilde{b}_{1}$ and $\tilde{b}_{2}$ are mixtures of lefthanded $(\mathrm{L})$ and right-handed $(\mathrm{R})$ bottom squarks, $\tilde{b}_{L}$ and $\tilde{b}_{R}$. The mixing is expressed as

$$
\begin{gathered}
\tilde{b}_{1}=\cos \theta_{\tilde{b}} \tilde{b}_{R}+\sin \theta_{\tilde{b}} \tilde{b}_{L}, \\
\tilde{b}_{2}=-\sin \theta_{\tilde{b}} \tilde{b}_{R}+\cos \theta_{\tilde{b}} \tilde{b}_{L} .
\end{gathered}
$$

In our notation, the lighter mass eigenstate is denoted $\tilde{b}_{1}$. For the case under consideration, the mixing of the bottom squark is determined by the condition that the lighter $\tilde{b}$ coupling to the $Z$ boson be small [2], namely $\sin \theta_{\tilde{b}} \simeq 0.38$.

Matrix Element and Decay Width. The coupling at the three-point vertex in which a $b$ quark enters and a $\tilde{b}_{1}$ squark emerges (the upper vertex in Fig. 1) is

$$
i g_{s} \sqrt{2} T_{j i}^{a}\left[\cos \theta_{\tilde{b}} P_{R}-\sin \theta_{\tilde{b}} P_{L}\right]
$$

where $i$ and $j$ are the color indices of the incident $b$ and final $\tilde{b}$, respectively, and $a$ labels the color of the exchanged gluino. $g_{s}$ is the strong coupling, $\alpha_{s}=g_{s}^{2} / 4 \pi$. Here, $P_{L}=\left(1-\gamma_{5}\right) / 2$ and $P_{R}=\left(1+\gamma_{5}\right) / 2$. At the lower vertex where an antiquark enters and $\overline{\tilde{b}}_{1}$ emerges, the coupling is

$$
i g_{s} \sqrt{2} T_{k \ell}^{a}\left[\cos \theta_{\tilde{b}} P_{L}-\sin \theta_{\tilde{b}} P_{R}\right]
$$


where $k$ and $\ell$ are the color indices of the incident $\bar{b}$ and final $\overline{\tilde{b}}$, respectively.

Define $t=\left(k_{1}-p_{\Upsilon} / 2\right)^{2}=-\left(m_{\Upsilon}^{2}-4 m_{1}^{2}\right) / 4$. The invariant amplitude for $\Upsilon \rightarrow \widetilde{b}\left(k_{1}\right)+\overline{\widetilde{b}}\left(k_{2}\right)$ is

$M=\frac{\sqrt{2} \pi \alpha_{s} A F_{c}}{t-m_{\tilde{g}}^{2}} \operatorname{Tr}\left(\not k\left(\not p_{\Upsilon}+m_{\Upsilon}\right)\left[\cos \theta_{\tilde{b}} P_{R}-\sin \theta_{\tilde{b}} P_{L}\right]\left[\not p_{\Upsilon}-2 \not k_{1}+2 m_{\tilde{g}}\right]\left[\cos \theta_{\tilde{b}} P_{L}-\sin \theta_{\tilde{b}} P_{R}\right]\right)$.

The color factor $F_{c}=\frac{4}{3 \sqrt{3}} \delta_{j k}$, where $j, k$ label the colors of the final squarks. The relatively large mass of the exchanged gluino should justify the use of simple perturbation theory to compute the decay amplitude. Evaluating the trace and using $\epsilon \cdot p_{\Upsilon}=0$, we obtain

$$
M=-\frac{4 \sqrt{2} \pi \alpha_{s} A m_{\Upsilon} F_{c}}{t-m_{\tilde{g}}^{2}} \epsilon \cdot k_{1}
$$

There is no explicit dependence on the mixing angle.

After squaring $M$, averaging over the spins of the $\Upsilon$, and summing over the colors of the final bottom squarks, we derive

$$
\Sigma|M|^{2}=-\frac{32 \cdot 16 \pi^{2}}{3 \cdot 9} \alpha_{s}^{2} A^{2} \frac{t m_{\Upsilon}^{2}}{\left(t-m_{\tilde{g}}^{2}\right)^{2}} .
$$

At threshold, $t \rightarrow 0$ and $|M| \rightarrow 0$.

To obtain the decay width of the $\Upsilon$ into $\tilde{b} \tilde{\bar{b}}$, we use the expression

$$
\Gamma=\frac{1}{8 \pi} \Sigma|M|^{2} \frac{|k|}{m_{\Upsilon}^{2}}
$$

where $|k|=\frac{1}{2} \sqrt{m_{\Upsilon}^{2}-4 m_{1}^{2}}$. We observe that $d \Gamma \propto|k|^{2 \ell+1}$, with $\ell=1$, as expected for the $p$-wave decay of the $J^{P}=1^{-} \Upsilon$ into a pair of scalars.

Predictions. Our results are presented in terms of a ratio

$$
R_{\tilde{b} \tilde{b}}^{\Upsilon}=\frac{\Gamma_{\tilde{b} \tilde{b}}}{\Gamma_{\ell \bar{\ell}}}=\frac{1}{3}\left(\frac{\alpha_{s}(\mu)}{\alpha_{\mathrm{em}}}\right)^{2} \frac{m_{\Upsilon}\left(m_{\Upsilon}^{2}-4 m_{\tilde{b}}^{2}\right)^{3 / 2}}{\left(t-m_{\tilde{g}}^{2}\right)^{2}},
$$

so that the absolute value of the normalization constant $A$ cancels. We use the value $\alpha_{s}(\mu)=$ $0.22 \pm 0.02$ determined from data on $\Upsilon$ decays and evaluated at the scale $\mu=m_{b}=4.75$ $\mathrm{GeV}$ [19]. This value of $\alpha_{s}(\mu)$ is consistent with determinations from other processes at other physical scales when all are plotted in common with the predicted $O\left(\alpha_{s}^{4}\right)$ QCD dependence on $\mu$, with $\alpha_{s}\left(M_{Z}\right)=0.1184$ and $\Lambda_{\overline{\mathrm{MS}}}^{(5)}=213 \mathrm{MeV}$ 《19].

The choice of a value of $\alpha_{s}$ smaller than 0.22 might be deemed more appropriate. The presence of a light gluino slows the running of $\alpha_{s}(\mu)$. Extrapolation from $\alpha_{s}\left(M_{Z}\right)$ to $\alpha_{s}\left(m_{b}\right)$ with inclusion of a gluino of mass $16 \mathrm{GeV}$ reduces $\alpha_{s}\left(m_{b}\right)$ to $\simeq 0.189$. The value $\alpha_{s}(9.45 \mathrm{GeV})=0.163 \pm 0.002 \pm 0.014$ is extracted from data on radiative decays of the $\Upsilon$ [20]. We comment below on the quantitative changes in our results from use of $\alpha_{s}=0.16$ rather 
than $\alpha_{s}=0.22$. A smaller value of $\alpha_{s}$ leads to a more generous range of possible values of $m_{\tilde{g}}$ and $m_{\tilde{b}}$.

As long as $m_{\tilde{g}}^{2}>|t|=\left(m_{\Upsilon}^{2}-4 m_{1}^{2}\right) / 4$, as is true for the parameter space of interest to us, the functional dependence of Eq. (13) shows that the ratio $R_{\tilde{b} \tilde{b}}^{\Upsilon}$ increases with $m_{\Upsilon}$. For a given bottom squark mass, larger values of $R_{\tilde{b} \tilde{b}}^{\Upsilon}$ correspond to smaller values of the gluino mass.

The values of $\Gamma_{\ell \bar{\ell}}(n S)$ listed in the Particle Data Group's [21] compilation are $1.32 \pm$ $0.04 \pm 0.03 \mathrm{keV}, 0.52 \pm 0.03 \pm 0.01 \mathrm{keV}, \sim 0.47 \mathrm{keV}$, and $0.248 \pm 0.031 \mathrm{keV}$, for $n=1-4$, respectively. The corresponding full widths $\Gamma^{\Upsilon}$ are $52.5 \pm 1.8 \mathrm{keV}, 44 \pm 7 \mathrm{keV}, 26.3 \pm 3.5$ $\mathrm{keV}$, and $14 \pm 5 \mathrm{MeV}$. A ratio $R_{\tilde{b} \tilde{b}}^{\Upsilon}=1$ would fall within a one standard deviation $(\sigma)$ change of $\Gamma^{\Upsilon}$ in all cases, and a ratio $R_{\tilde{b} \tilde{b}}^{\Upsilon}=10$ is close to or within $1 \sigma$ except for the $\Upsilon(1 S)$ where it corresponds to $25 \%$ of $\Gamma^{\Upsilon}(1 S)$. As a working hypothesis, we take $R_{\tilde{b} \tilde{b}}^{\Upsilon}=1$ to 10 as a definition of the range in which to find solutions for the bottom squark and gluino masses.

In Fig. 2, we present our results for $R_{\tilde{b} \tilde{b}}^{\Upsilon}$ as a function of $m_{\tilde{b}}$ and $m_{\tilde{g}}$. We show sets of curves for the choices $R_{\tilde{b} \tilde{b}}^{\Upsilon}=1$ and 10. Within each set, the curves correspond to $\Upsilon(n S)$ with $n=1-3$, with $n$ increasing from the lowest to uppermost curve. The lines can be read as predictions of the width for the corresponding values of bottom squark and gluino masses, or as lower limits on the sparticle masses given a known bound on $R_{\tilde{b} \tilde{b}}^{\Upsilon}$.

The requirement $R_{\tilde{b} \tilde{b}}^{\Upsilon} \leq 10$ can be satisfied readily within the range of values of the gluino and bottom squark masses favored in Ref. [1]. For example, with $R_{\tilde{b} \tilde{b}}^{\Upsilon} \leq 10, m_{\tilde{g}} \leq 16 \mathrm{GeV}$ is achieved for $m_{\tilde{b}} \geq 3.5,3.9$, and $4.2 \mathrm{GeV}$ at the $\Upsilon(n S)$ with $n=1-3$. The more restrictive condition $R_{\tilde{b} \tilde{b}}^{\Upsilon} \leq 1$ at the $\Upsilon(1 S)$, along with $m_{\tilde{g}} \leq 16 \mathrm{GeV}$, would demand that $m_{\tilde{b}} \geq 4.5$ $\mathrm{GeV}$, still within the range of acceptable solutions in Ref. [1]. While these conclusions are consistent with the range of proposed masses in Ref. [1], our analysis of $\Upsilon$ decays shows nevertheless that bounds on the ratio $R_{\tilde{b} \tilde{b}}^{\Upsilon}$ are potentially powerful for the establishment of lower bounds on masses. For example, as is evident in Fig. 2, at the $\Upsilon(1 S)$ the choice $m_{\tilde{b}}=3.0 \mathrm{GeV}$ would require $m_{\tilde{g}} \geq 18 \mathrm{GeV}$ or $\geq 32 \mathrm{GeV}$ if $R_{\tilde{b} \tilde{b}}^{\Upsilon} \leq 10$ or $\leq 1$, respectively.

Rather than focusing on the value of $R_{\tilde{b} \tilde{b}}^{\Upsilon}$, we may examine instead the implications of the additional SUSY contribution for the full width of the $\Upsilon$. Because full width of the $\Upsilon(1 S)$ is the one known most precisely, the most sensitive results on the possible exclusion of decays into a pair of $\widetilde{b}$ 's with low mass come from measurements at the $\Upsilon(1 S)$, as long as $m_{\Upsilon(1 S)}>2 m_{\tilde{b}}$. The hadronic width of the $\Upsilon$ is calculated in conventional QCD perturbation theory [17,18] from the three-gluon decay subprocess, $\Upsilon \rightarrow 3 g$, and, as indicated in Eq. (3), $\Gamma_{3 g} \propto \alpha_{s}^{3}$. The SUSY subprocess of interest here adds a new term to the hadronic width from $\Upsilon \rightarrow \tilde{b}+\overline{\tilde{b}}$. If this new subprocess is present but ignored in the analysis of the hadronic width, the true value of $\alpha_{s}(\mu)$ will be smaller than that extracted from a standard QCD fit by the factor $\left(1-\Gamma_{\text {SUSY }} / \Gamma_{3 g}\right)^{\frac{1}{3}}$. For a contribution from the $\tilde{b} \tilde{b}$ final state that is $25 \%$ of $\Gamma^{\Upsilon}(1 S)$ (i.e. $R_{\tilde{b} \tilde{b}}^{\Upsilon}=10$ ), the value of $\alpha_{s}$ extracted via Eq. (3) will be reduced by a factor of 0.9 , at the lower edge of the approximately $10 \%$ uncertainty band on the value [19] $\alpha_{s}=0.22 \pm 0.02$ used in our calculation. As remarked above, an even smaller value is extracted from data on radiative decays of the $\Upsilon$ [20]. From the perspective of this examination of $\Gamma^{\Upsilon}$, we deduce that a contribution from $\tilde{b} \tilde{b}$ decays as large as $25 \%$ of $\Gamma^{\Upsilon}(1 S)$ cannot be excluded. A thorough analysis would require the computation of next-to-leading order contributions in 
SUSY-QCD to both the $3 g$ and $\tilde{b} \overline{\tilde{b}}$ amplitudes and the appropriate evolution of $\alpha_{s}(\mu)$ with inclusion of a light gluino and a light bottom squark.

The data sample is largest at the $\Upsilon(4 S)$. However, for a fixed $m_{\tilde{g}}=14 \mathrm{GeV}$, the branching fraction into a pair of bottom squarks is about $10^{-3}$ for $m_{\tilde{b}}=2.5 \mathrm{GeV}$, and it drops to about $10^{-4}$ for $m_{\tilde{b}}=4.85 \mathrm{GeV}$, below current sensitivity.

Discussion and Conclusions. Our study of $\Upsilon$ decay to a pair of bottom squarks demonstrates that experimental limits on the ratio $R_{\tilde{b} \tilde{b}}^{\Upsilon}$ could establish significant new lower bounds on the masses of the gluino and bottom squark if $m_{\tilde{b}}<m_{\Upsilon} / 2$. In Ref. [1], a supersymmetrybased contribution is suggested as an explanation for the observed size of the bottom quark cross section and time-averaged $B^{0} \bar{B}^{0}$ mixing probability observed at hadron colliders. The proposal requires a bottom squark in the mass range 2.5 to $5 \mathrm{GeV}$ together with a gluino in the range 12 to $16 \mathrm{GeV}$. In this paper, we show that measurements of $R_{\tilde{b} \tilde{b}}^{\Upsilon}$ could further constrain these allowed regions markedly. For example, with $m_{\tilde{g}}<16 \mathrm{GeV}$, the very restrictive experimental bound $R_{\tilde{b} \tilde{b}}^{\Upsilon} \leq 1$ at the $\Upsilon(1 S)$ requires $m_{\tilde{b}}>4.5 \mathrm{GeV}$ when $\alpha_{s}=0.22$ is used in the computation (and $m_{\tilde{b}}>4.35 \mathrm{GeV}$ when $\alpha_{s}=0.16$ is used). With $m_{\tilde{g}}<16 \mathrm{GeV}$, the bound $R_{\tilde{b} \tilde{b}}^{\Upsilon} \leq 10$ at the $\Upsilon(n S)$ would require $m_{\tilde{b}}>3.5,3.9$, and $4.2 \mathrm{GeV}$, respectively, for $n=1-3$ and $\alpha_{s}=0.22$ (and $m_{\tilde{b}}>2.6,3.2$, and $3.5 \mathrm{GeV}$ for $\alpha_{s}=0.16$ ). The bound $R_{\tilde{b} \tilde{b}}^{\Upsilon} \leq 10$ implies that the contribution from the $\tilde{b} \tilde{\tilde{b}}$ final state is as much as $25 \%$ of $\Gamma^{\Upsilon}(1 S)$, consistent, within uncertainties, with the determination of $\alpha_{s}$ from $\Upsilon$ decays. All of these values of $m_{\tilde{b}}$ are acceptable from the perspective of Ref. [1] but tend to be towards the upper end of the proposed range. Gluinos of mass $12 \mathrm{GeV}$ or less would be incompatible with the restriction $R_{\tilde{b} \tilde{b}}^{\Upsilon}<10$ unless $m_{\tilde{b}}>4.2,4.55$, and $4.75 \mathrm{GeV}$ at the $\Upsilon(n S), n=1-3(3.9,4.25$, and $4.45 \mathrm{GeV}$ for $\left.\alpha_{s}=0.16\right)$. Gluinos of mass $>35 \mathrm{GeV}$, or so, are compatible $\left(R_{\tilde{b} \tilde{b}}^{\Upsilon} \leq 1\right)$ with current information on the total width of the $\Upsilon$ states for all $m_{\tilde{b}}>2.5 \mathrm{GeV}$.

The presence of a SUSY contribution to the total width $\Gamma^{\Upsilon}$ means that the value of $\alpha_{s}$ extracted from data will be reduced by the factor $\left(1-\Gamma_{\mathrm{SUSY}} / \Gamma_{3 g}\right)^{\frac{1}{3}}$. With a smaller $\alpha_{s}$, the contribution from the conventional $3 g$ decay is reduced, compensated by the SUSY contribution, so as to maintain $\Gamma^{\Upsilon}$. As remarked, use of a smaller value of $\alpha_{s}(\mu)$ in our calculation allows for a broader range of allowed values (i.e. smaller values) of $m_{\tilde{g}}$ and $m_{\tilde{b}}$. Evolution of $\alpha_{s}(\mu)$ from $\mu=m_{b}$ to $\mu=m_{Z}$ is slowed by the presence of a light gluino. Thus, consistency is achieved because the smaller $\alpha_{s}\left(m_{b}\right)$ that allows for a greater contribution from $\Upsilon \rightarrow \tilde{b} \tilde{b}$ can still be made compatible with the same $\alpha_{s}\left(m_{Z}\right)$.

A dedicated experimental investigation of the possible decay $\Upsilon \rightarrow \widetilde{\widetilde{b}}$ is warranted. Direct observation of the decay requires an understanding of the ways that the $\widetilde{b}$ 's may manifest themselves [1]. If the $\widetilde{b}$ is relatively stable, the $\widetilde{b}$ could pick up a light $\bar{u}$ or $\bar{d}$ and become a $\widetilde{B}^{-}$or $\widetilde{B}^{0}$ "mesino" with $J=1 / 2$, the superpartner of the $B$ meson. The mesino has baryon number zero but acts like a heavy $\bar{p}$ - perhaps detectable with a time-of-flight apparatus or via $d E / d x$ measurements. R-parity-violating and lepton-number-violating decay of the $\widetilde{b}$ into at least one lepton, disfavored by CLEO data [9], would imply final states with soft leptons. Possible baryon-number-violating R-parity-violating decays of the $\widetilde{b}$ are $\overline{\widetilde{b}} \rightarrow u+s$; $\rightarrow c+d$; and $\rightarrow c+s$. These final states of four light quarks might be distinguishable from conventional hadronic final states mediated by the three-gluon intermediate state. Studies of thrust and other event-shape variables or of exclusive final states may provide discrimination. 
$\Upsilon$ 's produced in $e^{+} e^{-}$annihilation are polarized transversely because of their coupling to the intermediate virtual photon. Correspondingly, the final $\widetilde{b}$ squarks from $\Upsilon \rightarrow \widetilde{\widetilde{b}}$ will have an angular distribution proportional to $\sin ^{2} \theta$.

A theoretical study similar to that in this paper may be carried out for decays of other bottomonium states into pairs of bottom squarks.

Acknowledgments. ELB is grateful to Tim Tait for clarifying discussions on squark mixing and to Brian Harris, David Kaplan, Zack Sullivan, Tim Tait, and Carlos Wagner for many valuable conversations. LC thanks Thomas Gajdosik for discussions on angular distributions. This research began during a lunchtime conversation at Frontiers in Contemporary Physics, Vanderbilt University, March 5 - 10, 2001. Work in the High Energy Physics Division at Argonne National Laboratory is supported by the U.S. Department of Energy, Division of High Energy Physics, Contract W-31-109-ENG-38. LC is partially supported under DOE Contract DE-FG92-96ER40967. 


\section{REFERENCES}

[1] E. L. Berger, B. W. Harris, D. E. Kaplan, Z. Sullivan, T. M. P. Tait, C. E. M. Wagner, Phys. Rev. Lett. 86, 4231 (2001); E. L. Berger, hep-ph/0103145, to be published in the Proceedings of the Fourth International Workshop on B Physics and CP Violation, Ise-Shima, Japan, February 18 - 23, 2001.

[2] M. Carena, S. Heinemeyer, C. E. M. Wagner, and G. Weiglein, Phys. Rev. Lett. 86, 4463 (2001).

[3] C. R. Nappi, Phys. Rev. D 25, 84 (1982); S. Pacetti and Y. Srivastava, hep-ph/0007318.

[4] A. Dedes and H. Dreiner, hep-ph/0009001.

[5] A. Arhrib, C.-K. Chua, and W.-S. Hou, hep-ph/0103094.

[6] DELPHI Collaboration, P. Abreu, et al. Phys. Lett. B444, 491 (1998) and Phys. Lett. B478, 65 (2000).

[7] BES Collaboration, J. Z. Bai, et al., hep-ex/0102003.

[8] CELLO Collaboration, H.-J. Behrend et al., Phys. Lett. B183, 400 (1987).

[9] CLEO Collaboration, V. Savinov et al., Phys. Rev. D 63, 051101 (2001).

[10] P. Fayet, Nucl. Phys. B90, 104 (1975).

[11] G. Farrar and P. Fayet, Phys. Lett. 76B, 575 (1978).

[12] L. Clavelli, "New Possibilities for a Light Gluino", Paper contributed to the LeptonPhoton Symposium, Stanford, 1999 (hep-ph/9908342).

[13] S. Raby, Phys. Rev. D 56, 2852 (1997) and Phys. Lett. B 422, 158 (1998); A. Mafi and S. Raby, hep-ph/9912436.

[14] J. H. Kühn, J. Kaplan, and E. Safiani, Nucl. Phys. B157, 125 (1979).

[15] E. L. Berger and D. Jones, Phys. Rev D 23, 1521 (1981) and Phys. Lett. 121B, 61 (1983).

[16] L. Clavelli, Phys. Rev. D 26, 1610 (1982).

[17] T. Appelquist and D. Politzer, Phys. Rev. Lett. 34, 43 (1975).

[18] R. Barbieri, G. Curci, E. d'Emilio, and E. Remiddi, Nucl. Phys. B154, 535 (1979); P. B. Mackenzie and G. P. Lepage, Phys. Rev. Lett. 47, 1244 (1981); J. H. Kühn, A. A. Penin, and A. A. Pivovarov, Nucl. Phys. B534, 356 (1998).

[19] S. Bethke J. Phys. G26, R27 (2000).

[20] CLEO Collaboration, B. Nemati et al., Phys. Rev. D 55, 5273 (1997).

[21] Particle Data Group, Eur. Phys. Jour. C15 1, (2000). 


\section{FIGURES}

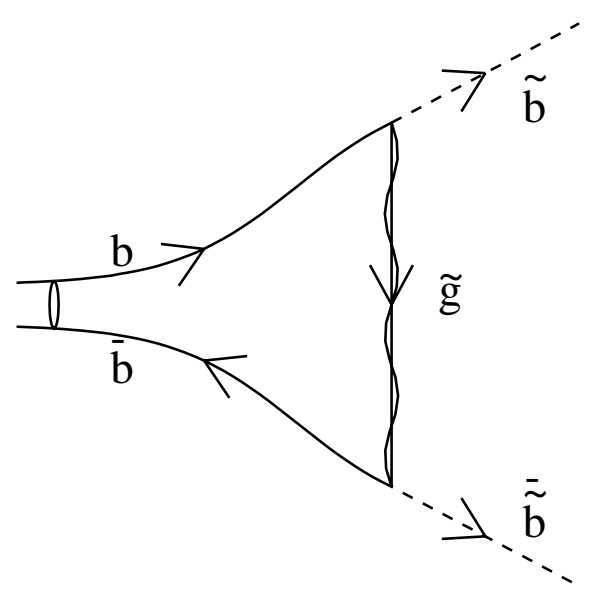

FIG. 1. Diagram of the decay of a bound $b \bar{b}$ system into a $\tilde{b} \bar{b}$ final state, mediated by single gluino exchange. 


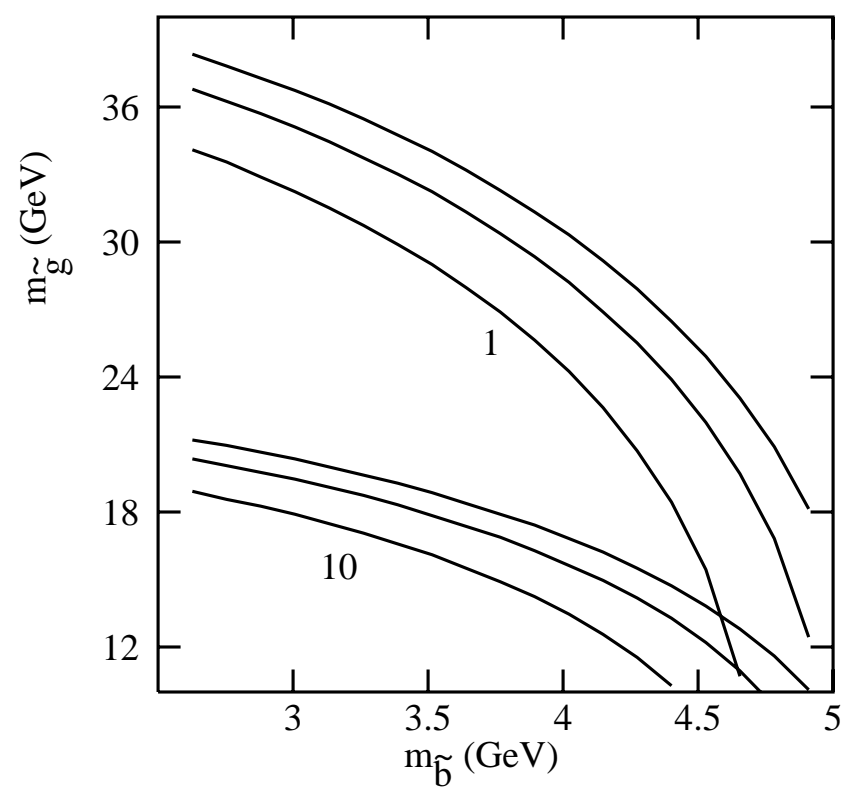

FIG. 2. Loci in the $m_{\tilde{b}}-m_{\tilde{g}}$ plane for which the rate for decay into a pair of bottom squarks is either 10 times $\Gamma_{\ell \bar{\ell}}$ for each $\Upsilon(n S)$, with $n=1-3$ (lower set of curves) or equal to $\Gamma_{\ell \bar{\ell}}$ (upper set). Above the curves, the rate would be less. Within each set of curves, the order is (bottom to top) $1 S, 2 S, 3 S$. 